\title{
Rutile Mineral Chemistry as a Guide to Provenance of Red Sediments and Modern Sands of Bhimunipatnam-Konada Coast, Andhra Pradesh, East Coast of India
}

\author{
K. Bangaku Naidu ${ }^{1}$ K. S. N. Reddy ${ }^{2}$ Ch. Ravi Sekhar ${ }^{2} \cdot$ P. Ganapati Rao ${ }^{3}$. \\ K. N. Murali Krishna ${ }^{4}$
}

Received: 5 August 2016/Revised: 29 June 2018/Accepted: 27 December 2018/Published online: 14 June 2019

(C) The Author(s) 2019

\begin{abstract}
Rutile is the most stable and widely distributed $\mathrm{TiO}_{2}$ polymorph in rocks of low- to high-grade metamorphic facies and is also an accessory mineral in igneous rocks. Rutile is commonly available in modern to ancient placer mineral deposits in the coastal sediments. Mineral chemistry of rutile from red sediments and modern sands along Bhimunipatnam-Konada coast were used in the present study to know its provenance. Iron $(\mathrm{Fe})$, chromium $(\mathrm{Cr})$, niobium $(\mathrm{Nb})$ content and their distribution pattern in rutile and also their relationships with aluminum (Al) and magnesium $(\mathrm{Mg})$ concentrations provide information on its provenance. These study reveals that the $\mathrm{Fe}-\mathrm{Cr}$ and $\mathrm{Cr}-\mathrm{Nb}$ systematics indicates majority of rutiles were derived from metapelitic rocks mainly khondalites and leptynites of the Eastern Ghats Granulite Belt (EGGB) and minor
\end{abstract}

K. Bangaku Naidu

naidu0756@gmail.com

K. S. N. Reddy

snrkurrys@reddifmail.com

Ch. Ravi Sekhar

ravisehargeo@gmail.com

P. Ganapati Rao

ganapathigeol@gmail.com

K. N. Murali Krishna

murali.dst2013@gmail.com

1 Center for Environment, Jawaharlal Nehru Technological University, Hyderabad 500085, Telangana, India

2 Department of Geology, Andhra University, Visakhapatnam 530003, Andhra Pradesh, India

3 Department of Geology, M.R.College (A), Vizianagaram 535002, Andhra Pradesh, India

4 SRKR Engineering College, Bhimavaram 534204, Andhra Pradesh, India contribution is from magmatic charnockites, pegmatites and granites. The $\mathrm{Al}$ and $\mathrm{Mg}$ behavioral pattern in rutile from both zones clearly depicts that the most of the rutiles are derived from crustal rocks. The rutile contribution to late quaternary red sediments and modern coastal sands is also from same provenance.

Keywords Rutile $\cdot$ Red sediments $\cdot$ Modern coastal sands · Khondalites · Charnockites

For the last few decades, recent development in mineralchemical provenance studies concerns advance in understanding of the mineral chemistry of rutile. Rutile mineral chemistry was used for genetic information [1]. Rutile predominantly forms in medium- to high-grade metamorphic rocks, although it has been recorded in plutonic rocks, such as granitoids and anorthosites [2]. It is scarce or absent in most of igneous and low-grade metamorphic rocks [3, 4]. The large variation in major elemental composition of rutile geochemistry is used in provenance studies. Detrital rutile in Triassic continental red-beds has been studied in beryl field [5, 6]. North Sea comprises almost pure $\mathrm{TiO}_{2}$, with only small proportion containing appreciable amount of $\mathrm{Nb}_{2} \mathrm{O}_{5}, \mathrm{Ta}_{2} \mathrm{O}_{5}$ or $\mathrm{FeO}$.

Electron probe microanalyzer data on rutile indicate that a large number of trace elements $(\mathrm{Fe}, \mathrm{Cr}, \mathrm{Al}, \mathrm{Mg}, \mathrm{Nb}, \mathrm{Ta})$ may substitute $\mathrm{Ti}$ in the rutile lattice [7-15] and the trace elements signature could be used as a provenance indicator. Detrital rutile geochemical studies by Meinhold et al., Stendal et al. and Triebold et al. [13, 16, 17] have demarcated the effectiveness of the method in identifying source and containing the metamorphic evolution of the hinterland. Rutiles were discriminated from crustal and mantle derived sources [10]. 
The chemical analysis of detrital rutiles may yield significant provenance information which is of particular importance because of stability of rutile under both burial diagenetic and surficial weathering conditions [18-20]. Previous investigators $[9,13,16,17,21]$ have been undertaken rutile geochemistry studies effectively identification for provenance.

This paper deals with detrital rutile geochemistry as guide to provenance of red sediments and modern sands of Bhimunipatnam-Konada coast, Andhra Pradesh, East Coast of India. In India, detrital rutile mineral chemical studies have been taken Kerala beach sands [22]. The occurrence of rutiles has been reported in charnockites, khondalites and pyroxene granulites of Eastern Ghat Group of rocks [23].

The present study area is bounded by longitude $83^{\circ} 23^{\prime}$ to $83^{\circ} 36^{\prime} \mathrm{E}$ and latitude $17^{\circ} 51^{\prime}$ to $18^{\circ} 02^{\prime} \mathrm{N}$. Major rivers flowing in the study area are Champavathi and Gosthani. These ephemeral rivers originate in the Eastern Ghat hill ranges, constitute the drainage system, carry huge amount of sediment, and debouch in to the Bay of Bengal at Bhimunipatnam and Konada in the study area. The coastal stretch of extending for $25 \mathrm{~km}$ the study area extends from the Gosthani River in the South to the Champavathi River in the North both are joins into Bay of Bengal. These ephemeral rivers originate in the Eastern Ghat hill ranges and constitute the drainage system and carry huge amount of sediment and debouch in to the Bay of Bengal at Bhimunipatnam and Konada in the study area. The study area has different geological and geomorphic features generated by the rivers, small creeks, altered coastal trends, and dynamic seasonal winds. In the study area, average width of coastal sand deposit is $980 \mathrm{~m}$ and dunes with maximum thickness of $18 \mathrm{~m}$. Predominantly redlooking sands covering an area of $10 \mathrm{~km}^{2}$ are popularly known as Bhimunipatnam "Red Sediments." These sands extend 1.5-2.5 km inland from the beach and $5 \mathrm{~km}$ along the coast at the $4 \mathrm{~km}$ south of Bhimunipatnam. Selected sample locations for provenance studies are given in Fig. 1A.

The Eastern Ghats Granulite Belt along the east coast of India extends for over $1000 \mathrm{~km}$. Major rock units in this granulite terrain are khondalites exhibiting compositional heterogeneity from sector to sector and charnockites [24] and lesser abundances of basic granulites, intrusive alkaline rocks, anorthosites [25], pyroxene granulites, syn-posttectonic granites [26], quartzites and leptynites.

During the study, a total of ten samples were collected from the red sediments and modern coastal sands. These collected samples were sun-dried, later representative sediment samples were reduced by coning and quartering method, heavy mineral separation was done by using bromoform, and the rutile mineral grains were identified under binocular petrological microscope based on their optical properties and picked for geochemical analysis (EPMA). Thirty rutile grains (twelve from yellow sand unit, six from reddish brown unit and twelve from brick red sand unit) from different sand units of red sediments and thirty rutile grains from modern sands of Bhimunipatnam-Konada coast were selected for chemical analysis.

The mineral grains were analyzed using a CAMECA SX-100 electron probe microanalyzer (EPMA), housed at the Geological Survey of India (GSI), Hyderabad. Polished surfaces of rutile grains were exited by an electron beam with an accelerating voltage of $15 \mathrm{kV}$ and the beam current $20 \mathrm{nA}$. The beam radius was kept at $\sim 1 \mu \mathrm{m}$. For calibration, natural mineral standards were used for most of the elements (Orthoclase for $\mathrm{Si}$ and $\mathrm{K}$; Corundum-Al; Wollastonite-Ca; Haematite-Fe; Apatite-P; Chromite-Cr; Albite for $\mathrm{Na}$ and $\mathrm{Al}$; Diopside for $\mathrm{Mg}$ and $\mathrm{Ca}$; Apatite-P; Rhodonite-Mn; $\mathrm{TiO}_{2}-\mathrm{Ti}$; Almandine-Fe; $\mathrm{Nb}$ on $\mathrm{Nb}$ and $\mathrm{Ta}$ on Ta). Mineral chemical data of detrital rutile from red sediments and modern sands of Bhimunipatnam-Konada coast are given in Table 1.

Rutile is one of the important economic heavy minerals for titanium, and it forms in high-grade metamorphic rocks such as granulites and eclogite facies of rocks. Rutile is widely distributed in many igneous and plutonic rocks, but placer deposits are the main source of extraction of rutile for economic purpose, and to understand its industrial suitability, geochemistry of rutiles was studied.

Rutile is one of the important stable detrital minerals in sedimentary environments and mainly consists of titanium dioxide $\left(\mathrm{TiO}_{2}\right)$ and important carrier of $\mathrm{Nb}, \mathrm{Cr}, \mathrm{Ta}, \mathrm{V}, \mathrm{Mo}$, $\mathrm{Sn}, \mathrm{Sb}$, and $\mathrm{W}$ elements [7, 27]. The information contained in rutile is most important to study the maturity of sediments. In recent times, many scientists made an attempt on provenance studies based on rutile mineral chemistry.

Heavy minerals are a significant tool to interpret the provenance, source of rock, transportation history of continental-beach-dune-shallow marine sediments [28].

The photomicrographs of rutile grains are red, blood red and reddish brown in color with rounded (a, b), anhedral (c, d) and prismatic (e, f) in shape (Fig. 1B a-f) grains with well-developed terminations or breakage patterns; sometimes thick halo surrounds the grains and very high relief and distinct pleochroism. Occasionally, striations are observed.

The rounded and sub-rounded grains of rutile indicate long distance of transportation and reworked nature. The sub-angular grains of rutiles indicate that they might have been derived from nearby sources, i.e., mainly khondalite and charnockites.

The $\mathrm{TiO}_{2}$ content of red sediments ranges from 97.68 to $99.78 \%$ (av. 98.66\%) and the $\mathrm{TiO}_{2}$ content of rutiles of 


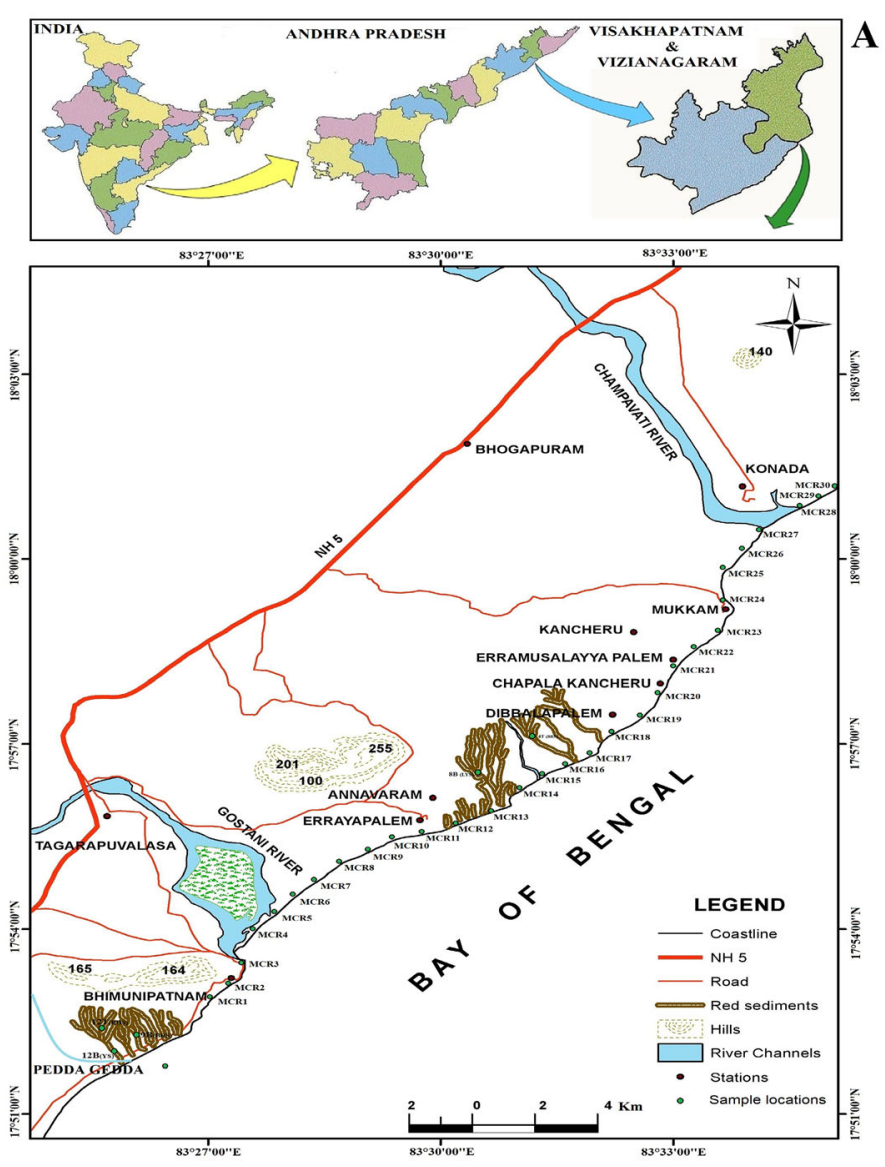

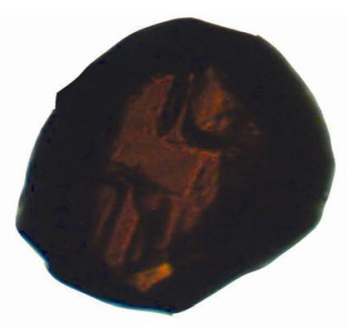

$\mathbf{a}$
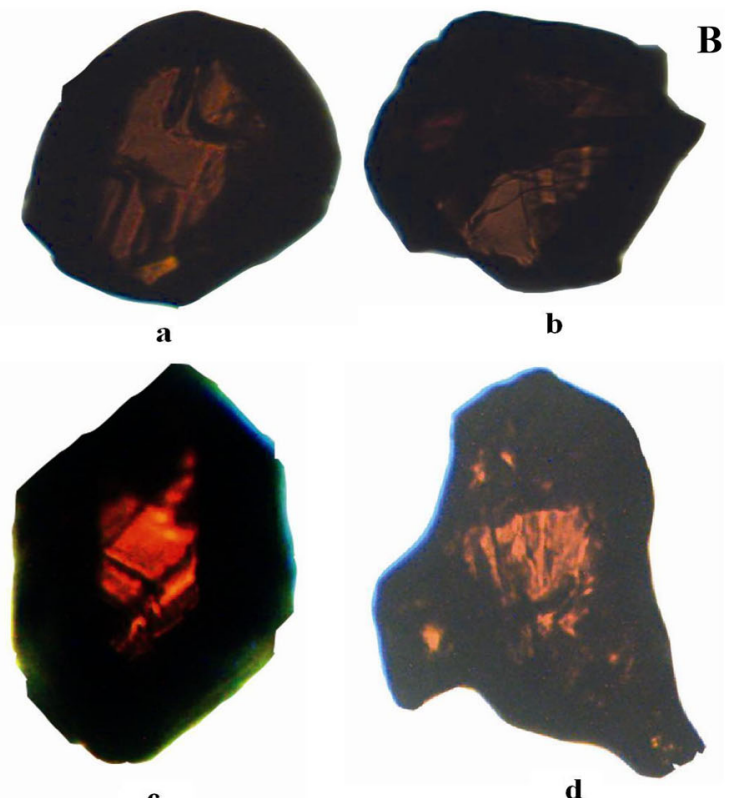

b
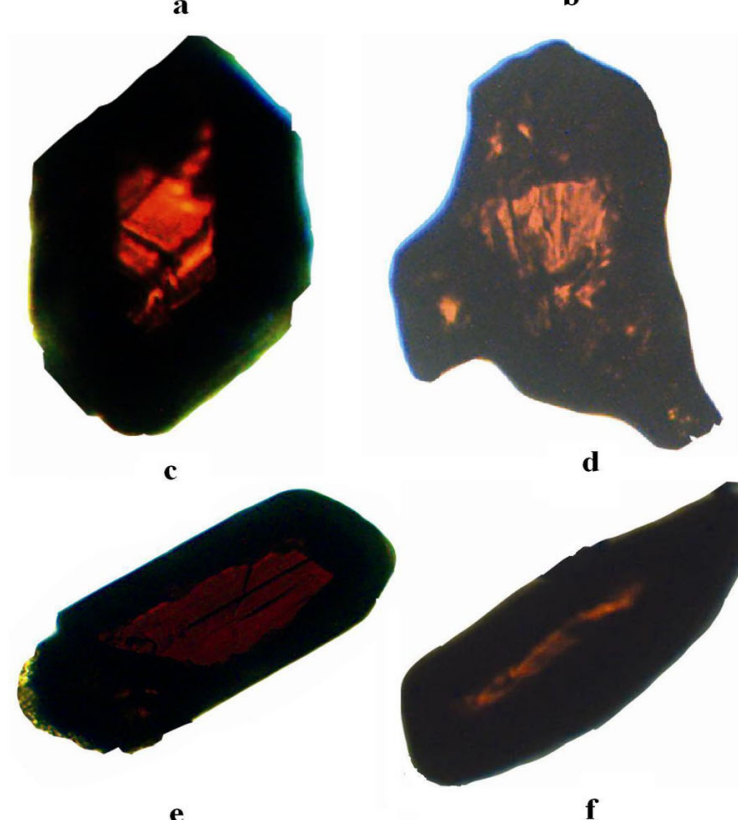

d

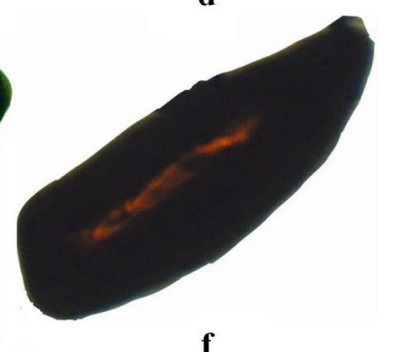

Fig. 1 A Map of the study area and $\mathbf{B}$ rutile grains (a-f)

modern sands of Bhimunipatnam-Konada coast ranges from 97.48 to $99.13 \%$ (av. $98.10 \%$ ).

The Fe content of rutiles of red sediments ranges from 70 to $2332 \mathrm{ppm}$ (av. $864.33 \mathrm{ppm}$ ). The Cr content of rutiles of red sediments ranges from 629 to $12302 \mathrm{ppm}$ (av. $2826 \mathrm{ppm})$. The $\mathrm{Nb}$ content of rutiles of red sediments ranges from 16 to $9664 \mathrm{ppm}$ (av. 3640).

The Fe content of rutiles of Bhimunipatnam-Konada modern coastal sands ranges from 8 to $1096 \mathrm{ppm}$ (av. $435 \mathrm{ppm}$ ). The $\mathrm{Cr}$ content of rutiles of modern sands ranges from 424 to $8402 \mathrm{ppm}$ (av. $2589 \mathrm{ppm}$ ). The $\mathrm{Nb}$ content of rutiles of modern sands ranges from 79 to $8551 \mathrm{ppm}$ (av. 3708 ppm).

$\mathrm{Cr}$ versus $\mathrm{Fe}$ systematics is not established thoroughly, but an attempt was made to differentiate rutiles based on $\mathrm{Fe}$ and $\mathrm{Cr}$ contents [9]. Rutiles with $\mathrm{Fe}$ content $<1000 \mathrm{ppm}$ and $\mathrm{Cr}$ content $>3000 \mathrm{ppm}$ are considered to be derived from magmatic rocks [9]. In order to understand provenance of rutiles of red sediments and modern coastal sands, the $\mathrm{Cr}$ versus $\mathrm{Fe}$ scatter plots and phi charts show $20 \%$ of analyzed samples of red sediment rutiles fall in magmatic zone, while remaining $80 \%$ fall in metamorphic zone
(Fig. 2a) and $17 \%$ of analyzed samples of modern coastal sand rutiles fall in magmatic zone and remaining $83 \%$ of analyzed samples of rutiles fall in metamorphic zone (Fig. 2b) of the study area.

The concentration of $\mathrm{Nb}$ and $\mathrm{Cr}$ in rutile can be used to distinguish rutile formed in metamafic and metapelitic rocks. The $\mathrm{Nb}$ content in metapelites ranges from 900 to 2700 ppm [29, 30]. Scatter plot of $\mathrm{Nb}$ versus $\mathrm{Cr}$ [21] was used to differentiate whether rutiles are derived from metapelitic rocks or from metamafic rocks.

$\mathrm{Nb}$ versus $\mathrm{Cr}$ scatter plot [21] was used to differentiate whether rutiles are derived from metapelitic rocks or from metamafic rocks. $\mathrm{Nb}$ versus $\mathrm{Cr}$ diagram and phi charts (Fig. 2a) show $71 \%$ of analyzed samples of red sediments, indicating that they are derived from metapelitic rocks and remaining 29\% from metamafic rocks, and modern coastal sands of $\mathrm{Nb}$ versus $\mathrm{Cr}$ diagram (Fig. 2b) show 64\% of analyzed samples of red sediments, indicating that they are derived from metapelitic rocks and remaining 36\% from metamafic rocks; both show mixed rutile source with more pronounced metapelitic rocks and minor contribution from metamafic rocks of the study area. 
Table 1 Chemical composition of rutiles from red sediments and modern coastal sands of Bhimunipatnam-Konada coast (Wt\%)

\begin{tabular}{|c|c|c|c|c|c|c|c|c|c|c|c|c|c|c|c|}
\hline & MCR1 & MCR2 & MCR3 & MCR4 & MCR5 & MCR6 & MCR7 & MCR8 & MCR9 & MCR10 & MCR11 & MCR12 & MCR13 & MCR14 & MCR15 \\
\hline $\mathrm{SiO}_{2}$ & 0.02 & 0.01 & 0.00 & 0.21 & 0.02 & 0.05 & 0.00 & 0.02 & 0.01 & 0.04 & 0.01 & 0.02 & 0.00 & 0.01 & 0.03 \\
\hline $\mathrm{TiO}_{2}$ & 97.48 & 97.68 & 98.26 & 97.61 & 98.24 & 98.91 & 97.98 & 98.21 & 98.26 & 98.48 & 97.98 & 97.75 & 98.35 & 98.65 & 97.74 \\
\hline $\mathrm{Al}_{2} \mathrm{O}_{3}$ & 0.55 & 0.06 & 0.01 & 0.00 & 0.01 & 0.00 & 0.03 & 0.03 & 0.03 & 0.01 & 0.01 & 0.03 & 0.01 & 0.01 & 0.01 \\
\hline $\mathrm{FeO}$ & 0.04 & 0.08 & 0.07 & 0.09 & 0.13 & 0.22 & 0.11 & 0.03 & 0.11 & 0.03 & 0.02 & 0.00 & 0.01 & 0.03 & 0.07 \\
\hline $\mathrm{MnO}$ & 0.02 & 0.01 & 0.02 & 0.00 & 0.02 & 0.00 & 0.01 & 0.01 & 0.01 & 0.01 & 0.01 & 0.02 & 0.00 & 0.09 & 0.02 \\
\hline $\mathrm{MgO}$ & 0.00 & 0.01 & 0.01 & 0.14 & 0.01 & 0.03 & 0.00 & 0.02 & 0.01 & 0.00 & 0.02 & 0.03 & 0.01 & 0.00 & 0.00 \\
\hline $\mathrm{CaO}$ & 0.04 & 0.01 & 0.02 & 0.03 & 0.01 & 0.04 & 0.01 & 0.03 & 0.03 & 0.02 & 0.00 & 0.03 & 0.01 & 0.02 & 0.01 \\
\hline $\mathrm{Na}_{2} \mathrm{O}$ & 0.03 & 0.01 & 0.01 & 0.02 & 0.01 & 0.03 & 0.01 & 0.02 & 0.02 & 0.02 & 0.01 & 0.00 & 0.01 & 0.02 & 0.01 \\
\hline $\mathrm{K}_{2} \mathrm{O}$ & 0.01 & 0.01 & 0.02 & 0.01 & 0.03 & 0.01 & 0.00 & 0.00 & 0.02 & 0.00 & 0.01 & 0.02 & 0.02 & 0.01 & 0.00 \\
\hline $\mathrm{Cr}_{2} \mathrm{O}_{3}$ & 0.15 & 0.04 & 0.21 & 0.11 & 0.07 & 0.09 & 0.19 & 0.27 & 0.14 & 0.16 & 0.61 & 0.18 & 0.19 & 0.34 & 0.24 \\
\hline $\mathrm{Nb}_{2} \mathrm{O}_{3}$ & 0.26 & 0.23 & 0.01 & 0.13 & 0.16 & 0.41 & 0.34 & 0.32 & 0.34 & 0.19 & 0.22 & 0.41 & 0.23 & 0.21 & 0.07 \\
\hline $\mathrm{Ta}_{2} \mathrm{O} 5$ & 0.04 & 0.04 & 0.03 & 0.04 & 0.03 & 0.00 & 0.00 & 0.04 & 0.00 & 0.04 & 0.02 & 0.04 & 0.03 & 0.01 & 0.07 \\
\hline $\mathrm{P}_{2} \mathrm{O}_{5}$ & 0.01 & 0.00 & 0.00 & 0.04 & 0.01 & 0.05 & 0.03 & 0.00 & 0.02 & 0.01 & 0.02 & 0.05 & 0.04 & 0.05 & 0.02 \\
\hline Total & 98.66 & 98.19 & 98.66 & 98.44 & 98.77 & 99.85 & 98.71 & 98.99 & 98.99 & 99.01 & 98.95 & 98.57 & 98.89 & 99.46 & 98.30 \\
\hline \multicolumn{16}{|c|}{ Number of cations on the basis of $4(O)$} \\
\hline $\mathrm{Si}$ & 0.00 & 0.00 & 0.00 & 0.01 & 0.00 & 0.00 & 0.00 & 0.00 & 0.00 & 0.00 & 0.00 & 0.00 & 0.00 & 0.00 & 0.00 \\
\hline $\mathrm{Al}$ & 0.02 & 0.00 & 0.00 & 0.00 & 0.00 & 0.00 & 0.00 & 0.00 & 0.00 & 0.00 & 0.00 & 0.00 & 0.00 & 0.00 & 0.00 \\
\hline $\mathrm{Ti}$ & 1.98 & 1.99 & 1.99 & 1.98 & 1.99 & 1.99 & 1.99 & 1.99 & 1.99 & 1.99 & 1.98 & 1.99 & 1.99 & 1.99 & 1.99 \\
\hline $\mathrm{Fe}$ & 0.00 & 0.00 & 0.00 & 0.00 & 0.00 & 0.00 & 0.00 & 0.00 & 0.00 & 0.00 & 0.00 & 0.00 & 0.00 & 0.00 & 0.00 \\
\hline $\mathrm{Mn}$ & 0.00 & 0.00 & 0.00 & 0.00 & 0.00 & 0.00 & 0.00 & 0.00 & 0.00 & 0.00 & 0.00 & 0.00 & 0.00 & 0.00 & 0.00 \\
\hline $\mathrm{Mg}$ & 0.00 & 0.00 & 0.00 & 0.01 & 0.00 & 0.00 & 0.00 & 0.00 & 0.00 & 0.00 & 0.00 & 0.00 & 0.00 & 0.00 & 0.00 \\
\hline $\mathrm{Ca}$ & 0.00 & 0.00 & 0.00 & 0.00 & 0.00 & 0.00 & 0.00 & 0.00 & 0.00 & 0.00 & 0.00 & 0.00 & 0.00 & 0.00 & 0.00 \\
\hline $\mathrm{Na}$ & 0.00 & 0.00 & 0.00 & 0.00 & 0.00 & 0.00 & 0.00 & 0.00 & 0.00 & 0.00 & 0.00 & 0.00 & 0.00 & 0.00 & 0.00 \\
\hline $\mathrm{K}$ & 0.00 & 0.00 & 0.00 & 0.00 & 0.00 & 0.00 & 0.00 & 0.00 & 0.00 & 0.00 & 0.00 & 0.00 & 0.00 & 0.00 & 0.00 \\
\hline $\mathrm{Cr}$ & 0.00 & 0.00 & 0.00 & 0.00 & 0.00 & 0.00 & 0.00 & 0.01 & 0.00 & 0.00 & 0.01 & 0.00 & 0.00 & 0.01 & 0.01 \\
\hline $\mathrm{Nb}$ & 0.00 & 0.00 & 0.00 & 0.00 & 0.00 & 0.00 & 0.00 & 0.00 & 0.00 & 0.00 & 0.00 & 0.00 & 0.00 & 0.00 & 0.00 \\
\hline Тa & 0.00 & 0.00 & 0.00 & 0.00 & 0.00 & 0.00 & 0.00 & 0.00 & 0.00 & 0.00 & 0.00 & 0.00 & 0.00 & 0.00 & 0.00 \\
\hline $\mathrm{P}$ & 0.00 & 0.00 & 0.00 & 0.00 & 0.00 & 0.00 & 0.00 & 0.00 & 0.00 & 0.00 & 0.00 & 0.00 & 0.00 & 0.00 & 0.00 \\
\hline \multirow[t]{2}{*}{ Total } & 2.01 & 2.00 & 2.00 & 2.01 & 2.00 & 2.00 & 2.00 & 2.00 & 2.00 & 2.00 & 2.00 & 2.00 & 2.00 & 2.00 & 2.00 \\
\hline & MCR16 & MCR17 & MCR18 & MCR19 & MCR20 & MCR21 & MCR22 & 2 MCR23 & 3 MCR24 & $4 \quad$ MCR25 & MCR26 & MCR27 & MCR28 & MCR29 & MCR30 \\
\hline $\mathrm{SiO}_{2}$ & 0.02 & 0.01 & 0.02 & 0.05 & 0.04 & 0.01 & 0.03 & 0.00 & 0.05 & 0.02 & 0.01 & 0.01 & 0.03 & 0.01 & 0.00 \\
\hline $\mathrm{TiO}_{2}$ & 97.80 & 97.83 & 98.40 & 97.58 & 97.72 & 98.14 & 99.13 & 98.34 & 97.71 & 98.43 & 97.91 & 98.03 & 98.41 & 97.73 & 98.31 \\
\hline $\mathrm{Al}_{2} \mathrm{O}_{3}$ & 0.01 & 0.02 & 0.01 & 0.01 & 0.07 & 0.01 & 0.01 & 0.02 & 0.03 & 0.03 & 0.04 & 0.01 & 0.00 & 0.02 & 0.02 \\
\hline $\mathrm{FeO}$ & 0.04 & 0.07 & 0.03 & 0.14 & 0.11 & 0.04 & 0.02 & 0.04 & 0.11 & 0.08 & 0.23 & 0.11 & 0.17 & 0.14 & 0.07 \\
\hline $\mathrm{MnO}$ & 0.00 & 0.04 & 0.01 & 0.00 & 0.01 & 0.02 & 0.03 & 0.09 & 0.02 & 0.00 & 0.01 & 0.01 & 0.06 & 0.03 & 0.04 \\
\hline $\mathrm{MgO}$ & 0.02 & 0.00 & 0.02 & 0.01 & 0.01 & 0.00 & 0.00 & 0.03 & 0.01 & 0.02 & 0.01 & 0.00 & 0.00 & 0.02 & 0.02 \\
\hline $\mathrm{CaO}$ & 0.01 & 0.04 & 0.02 & 0.01 & 0.01 & 0.02 & 0.00 & 0.01 & 0.01 & 0.01 & 0.03 & 0.02 & 0.03 & 0.01 & 0.01 \\
\hline $\mathrm{Na}_{2} \mathrm{O}$ & 0.00 & 0.01 & 0.01 & 0.04 & 0.01 & 0.01 & 0.03 & 0.02 & 0.00 & 0.02 & 0.01 & 0.01 & 0.00 & 0.02 & 0.01 \\
\hline $\mathrm{K}_{2} \mathrm{O}$ & 0.02 & 0.00 & 0.00 & 0.02 & 0.01 & 0.02 & 0.00 & 0.00 & 0.00 & 0.01 & 0.01 & 0.02 & 0.01 & 0.00 & 0.02 \\
\hline $\mathrm{Cr}_{2} \mathrm{O}_{3}$ & 0.18 & 0.09 & 0.10 & 0.14 & 0.11 & 0.20 & 0.03 & 0.06 & 0.16 & 0.12 & 0.10 & 0.29 & 0.12 & 0.21 & 0.19 \\
\hline $\mathrm{Nb}_{2} \mathrm{O}_{3}$ & 0.13 & 0.01 & 0.07 & 0.22 & 0.54 & 0.43 & 0.09 & 0.12 & 0.26 & 0.25 & 0.36 & 0.45 & 0.07 & 0.53 & 0.20 \\
\hline $\mathrm{Ta}_{2} \mathrm{O}_{5}$ & 0.06 & 0.01 & 0.09 & 0.00 & 0.04 & 0.00 & 0.03 & 0.07 & 0.02 & 0.00 & 0.06 & 0.10 & 0.02 & 0.03 & 0.02 \\
\hline $\mathrm{P}_{2} \mathrm{O}_{5}$ & 0.02 & 0.04 & 0.00 & 0.05 & 0.02 & 0.01 & 0.01 & 0.03 & 0.00 & 0.03 & 0.00 & 0.03 & 0.01 & 0.04 & 0.03 \\
\hline Total & 98.29 & 98.16 & 98.78 & 98.26 & 98.68 & 98.90 & 99.41 & 98.83 & 98.39 & 99.03 & 98.78 & 99.09 & 98.92 & 98.79 & 98.94 \\
\hline \multicolumn{16}{|c|}{ Number of cations on the basis of $4(O)$} \\
\hline $\mathrm{Si}$ & 0.00 & 0.00 & 0.00 & 0.00 & 0.00 & 0.00 & 0.00 & 0.00 & 0.00 & 0.00 & 0.00 & 0.00 & 0.00 & 0.00 & 0.00 \\
\hline $\mathrm{Al}$ & 0.00 & 0.00 & 0.00 & 0.00 & 0.00 & 0.00 & 0.00 & 0.00 & 0.00 & 0.00 & 0.00 & 0.00 & 0.00 & 0.00 & 0.00 \\
\hline $\mathrm{Ti}$ & 1.99 & 1.99 & 1.99 & 1.99 & 1.98 & 1.99 & 2.00 & 1.99 & 1.99 & 1.99 & 1.99 & 1.98 & 1.99 & 1.98 & 1.99 \\
\hline $\mathrm{Fe}$ & 0.00 & 0.00 & 0.00 & 0.00 & 0.00 & 0.00 & 0.00 & 0.00 & 0.00 & 0.00 & 0.01 & 0.00 & 0.00 & 0.00 & 0.00 \\
\hline $\mathrm{Mn}$ & 0.00 & 0.00 & 0.00 & 0.00 & 0.00 & 0.00 & 0.00 & 0.00 & 0.00 & 0.00 & 0.00 & 0.00 & 0.00 & 0.00 & 0.00 \\
\hline $\mathrm{Mg}$ & 0.00 & 0.00 & 0.00 & 0.00 & 0.00 & 0.00 & 0.00 & 0.00 & 0.00 & 0.00 & 0.00 & 0.00 & 0.00 & 0.00 & 0.00 \\
\hline $\mathrm{Ca}$ & 0.00 & 0.00 & 0.00 & 0.00 & 0.00 & 0.00 & 0.00 & 0.00 & 0.00 & 0.00 & 0.00 & 0.00 & 0.00 & 0.00 & 0.00 \\
\hline
\end{tabular}


Table 1 continued

\begin{tabular}{|c|c|c|c|c|c|c|c|c|c|c|c|c|c|c|c|}
\hline & MCR16 & MCR17 & MCR18 & MCR19 & MCR20 & MCR21 & MCR22 & MCR23 & MCR24 & MCR25 & MCR26 & MCR27 & MCR28 & MCR29 & MCR30 \\
\hline $\mathrm{Na}$ & 0.00 & 0.00 & 0.00 & 0.00 & 0.00 & 0.00 & 0.00 & 0.00 & 0.00 & 0.00 & 0.00 & 0.00 & 0.00 & 0.00 & 0.00 \\
\hline K & 0.00 & 0.00 & 0.00 & 0.00 & 0.00 & 0.00 & 0.00 & 0.00 & 0.00 & 0.00 & 0.00 & 0.00 & 0.00 & 0.00 & 0.00 \\
\hline $\mathrm{Cr}$ & 0.00 & 0.00 & 0.00 & 0.00 & 0.00 & 0.00 & 0.00 & 0.00 & 0.00 & 0.00 & 0.00 & 0.01 & 0.00 & 0.00 & 0.00 \\
\hline $\mathrm{Nb}$ & 0.00 & 0.00 & 0.00 & 0.00 & 0.01 & 0.01 & 0.00 & 0.00 & 0.00 & 0.00 & 0.00 & 0.01 & 0.00 & 0.01 & 0.00 \\
\hline $\mathrm{Ta}$ & 0.00 & 0.00 & 0.00 & 0.00 & 0.00 & 0.00 & 0.00 & 0.00 & 0.00 & 0.00 & 0.00 & 0.00 & 0.00 & 0.00 & 0.00 \\
\hline $\mathrm{P}$ & 0.00 & 0.00 & 0.00 & 0.00 & 0.00 & 0.00 & 0.00 & 0.00 & 0.00 & 0.00 & 0.00 & 0.00 & 0.00 & 0.00 & 0.00 \\
\hline Total & 2.00 & 2.00 & 2.00 & 2.00 & 2.00 & 2.00 & 2.00 & 2.00 & 2.00 & 2.00 & 2.00 & 2.00 & 2.00 & 2.00 & 2.00 \\
\hline
\end{tabular}

\begin{tabular}{|c|c|c|c|c|c|c|c|c|c|c|c|c|c|c|c|c|c|c|}
\hline \multirow{2}{*}{$\begin{array}{l}\text { Sample no } \\
\text { Grain no }\end{array}$} & \multicolumn{6}{|c|}{$12 \mathrm{~T}$ (reddish brown sands) } & \multicolumn{6}{|c|}{ 4T (brick red sands) } & \multicolumn{6}{|c|}{ 9B (brick red sands) } \\
\hline & RBI & RB2 & RB3 & RB4 & RB5 & RB6 & $\mathrm{BR} 1$ & BR2 & BR3 & $\mathrm{BR} 4$ & BR5 & BR6 & BR1 & $\mathrm{BR} 2$ & BR3 & $\mathrm{BR} 4$ & BR5 & BR6 \\
\hline $\mathrm{SiO}_{2}$ & 0.00 & 0.01 & .00 & 0.01 & 0.03 & 0.02 & .00 & .00 & 0.01 & 0.02 & 0.01 & 0.05 & .03 & 0.00 & 0.01 & 0.01 & 0.02 & 0.03 \\
\hline $\mathrm{TiO}_{2}$ & 98.60 & 99.29 & 98.76 & 99.10 & 98.31 & 99.78 & 98.91 & 98.96 & 98.91 & 98.81 & 99.27 & 97.99 & 98.80 & 98.47 & 99.60 & 98.83 & 98.11 & 98.81 \\
\hline $\mathrm{Al}_{2} \mathrm{O}_{3}$ & 0.00 & 0.05 & 0.04 & 0.01 & 0.02 & 0.03 & 0.03 & 0.00 & 0.04 & 0.01 & 0.03 & 0.03 & 0.00 & 0.03 & 0.01 & 0.00 & 0.01 & 0.02 \\
\hline $\mathrm{FeO}$ & 0.05 & 0.20 & 0.18 & 0.03 & 0.30 & 0.14 & 0.11 & 0.08 & 0.11 & 0.05 & 0.08 & 0.23 & 0.23 & 0.01 & 0.13 & 0.14 & 0.13 & 0.01 \\
\hline $\mathrm{MnO}$ & 0.06 & 0.05 & 0.02 & 0.01 & 0.02 & 0.01 & 0.00 & 0.07 & 0.01 & 0.01 & 0.04 & 0.09 & 0.05 & 0.01 & 0.02 & 0.03 & 0.00 & 0.03 \\
\hline $\mathrm{MgO}$ & 0.01 & 0.02 & 0.01 & 0.01 & 0.01 & 0.01 & 0.03 & 0.01 & 0.00 & 0.02 & 0.01 & 0.01 & 0.02 & 0.00 & 0.02 & 0.01 & 0.01 & 0.02 \\
\hline $\mathrm{CaO}$ & 0.01 & 0.01 & 0.03 & 0.01 & 0.02 & 0.01 & 0.03 & 0.01 & 0.01 & 0.02 & 0.05 & 0.01 & 0.00 & 0.02 & 0.03 & 0.00 & 0.01 & 0.04 \\
\hline $\mathrm{Na}_{2} \mathrm{O}$ & 0.01 & 0.01 & 0.01 & 0.02 & 0.01 & 0.00 & 0.01 & 0.02 & 0.02 & 0.02 & 0.03 & 0.02 & 0.01 & 0.02 & 0.00 & 0.04 & 0.02 & 0.02 \\
\hline $\mathrm{K}_{2} \mathrm{O}$ & 0.01 & 0.01 & 0.00 & 0.02 & 0.00 & 0.01 & 0.01 & 0.01 & 0.01 & 0.01 & 0.01 & 0.01 & 0.01 & 0.02 & 0.01 & 0.01 & 0.04 & 0.01 \\
\hline $\mathrm{Cr}_{2} \mathrm{O}_{3}$ & 0.20 & 0.16 & 0.09 & 0.23 & 0.14 & 0.09 & 0.10 & 0.15 & 0.21 & 0.07 & 0.06 & 0.77 & 0.05 & 0.19 & 0.09 & 0.21 & 0.20 & 0.17 \\
\hline $\mathrm{Nb}_{2} \mathrm{O}_{3}$ & 0.25 & 0.38 & 0.22 & 0.05 & 0.44 & 0.05 & 0.15 & 0.17 & 0.32 & 0.03 & 0.20 & 0.50 & 0.09 & 0.44 & 0.35 & 0.24 & 0.11 & 0.27 \\
\hline $\mathrm{Ta}_{2} \mathrm{O}_{5}$ & 0.03 & 0.05 & 0.04 & 0.05 & 0.03 & 0.01 & 0.00 & 0.06 & 0.03 & 0.05 & 0.04 & 0.14 & 5 & 0.04 & 0.01 & 0.02 & 0.06 & 0.07 \\
\hline $\mathrm{P}_{2} \mathrm{O}_{5}$ & 0.01 & 0.03 & 0.02 & 0.05 & 0.03 & 0.00 & 0.00 & 0.01 & 0.02 & 0.01 & 0.02 & 0.03 & 0.00 & 0.01 & 0.02 & 0.00 & 0.03 & 0.01 \\
\hline Total & 99.24 & 100.27 & 99.42 & 99.6 & 99.36 & 100.16 & 99.38 & 99.55 & 99.7 & 99.13 & 99.85 & 99.88 & 99.34 & 99.26 & 100.3 & 99.54 & 98.75 & 99.51 \\
\hline \multicolumn{19}{|c|}{ Structural formulae normalized to 4 oxygens } \\
\hline $\mathrm{Si}$ & 0.00 & 0.00 & 0.00 & 0.00 & 0.00 & 0.00 & 0.00 & 0.00 & 0.00 & 0.00 & 0.00 & 0.00 & 0.00 & 0.00 & 0.00 & 0.00 & 0.00 & 0.00 \\
\hline $\mathrm{Al}$ & 0.00 & 0.00 & 0.00 & 0.00 & 0.00 & 0.00 & 0.00 & 0.00 & 0.00 & 0.00 & 0.00 & 0.00 & 0.00 & 0.00 & 0.00 & 0.00 & 0.00 & 0.00 \\
\hline $\mathrm{Ti}$ & 1.99 & 1.99 & 1.99 & 1.99 & 1.98 & 1.99 & 1.99 & 1.99 & 1.99 & 2.00 & 1.99 & 1.97 & 1.99 & 1.99 & 1.99 & 1.99 & 1.99 & 1.99 \\
\hline $\mathrm{Fe}$ & 0.00 & 0.00 & 0.00 & 0.00 & 0.01 & 0.00 & 0.00 & 0.00 & 0.00 & 0.00 & 0.00 & 0.01 & 0.01 & 0.00 & 0.00 & 0.00 & 0.00 & 0.00 \\
\hline $\mathrm{Mn}$ & 0.00 & 0.00 & 0.00 & 0.00 & 0.00 & 0.00 & 0.00 & 0.00 & 0.00 & 0.00 & 0.00 & 0.00 & 0.00 & 0.00 & 0.00 & 0.00 & 0.00 & 0.00 \\
\hline $\mathrm{Mg}$ & 0.00 & 0.00 & 0.00 & 0.00 & 0.00 & 0.00 & 0.00 & 0.00 & 0.00 & 0.00 & 0.00 & 0.00 & 0.00 & 0.00 & 0.00 & 0.00 & 0.00 & 0.00 \\
\hline $\mathrm{Ca}$ & 0.00 & 0.00 & 0.00 & 0.00 & 0.00 & 0.00 & 0.00 & 0.00 & 0.00 & 0.00 & 0.00 & 0.00 & 0.00 & 0.00 & 0.00 & 0.00 & 0.00 & 0.00 \\
\hline $\mathrm{Na}$ & 0.00 & 0.00 & 0.00 & 0.00 & 0.00 & 0.00 & 0.00 & 0.00 & 0.00 & 0.00 & 0.00 & 0.00 & 0.00 & 0.00 & 0.00 & 0.00 & 0.00 & 0.00 \\
\hline K & 0.00 & 0.00 & 0.00 & 0.00 & 0.00 & 0.00 & 0.00 & 0.00 & 0.00 & 0.00 & 0.00 & 0.00 & 0.00 & 0.00 & 0.00 & 0.00 & 0.00 & 0.00 \\
\hline $\mathrm{Cr}$ & 0.00 & 0.00 & 0.00 & 0.00 & 0.00 & 0.00 & 0.00 & 0.00 & 0.00 & 0.00 & 0.00 & 0.02 & 0.00 & 0.00 & 0.00 & 0.00 & 0.00 & 0.00 \\
\hline $\mathrm{Nb}$ & 0.00 & 0.00 & 0.00 & 0.00 & 0.01 & 0.00 & 0.00 & 0.00 & 0.00 & 0.00 & 0.00 & 0.01 & 0.00 & 0.01 & 0.00 & 0.00 & 0.00 & 0.00 \\
\hline $\mathrm{Ta}$ & 0.00 & 0.00 & 0.00 & 0.00 & 0.00 & 0.00 & 0.00 & 0.00 & 0.00 & 0.00 & 0.00 & 0.00 & 0.00 & 0.00 & 0.00 & 0.00 & 0.00 & 0.00 \\
\hline $\mathrm{P}$ & 0.00 & 0.00 & 0.00 & 0.00 & 0.00 & 0.00 & 0.00 & 0.00 & 0.00 & 0.00 & 0.00 & 0.00 & 0.00 & 0.00 & 0.00 & 0.00 & 0.00 & 0.00 \\
\hline Total & 2.00 & 2.00 & 2.00 & 2.00 & 2.00 & 2.00 & 2.00 & 2.00 & 2.00 & 2.00 & 2.00 & 2.01 & 2.00 & 2.00 & 2.00 & 2.00 & 2.00 & 2.00 \\
\hline
\end{tabular}

\begin{tabular}{|c|c|c|c|c|c|c|c|c|c|c|c|c|}
\hline \multirow{2}{*}{$\begin{array}{l}\text { Sample no. } \\
\text { Grain no. }\end{array}$} & \multicolumn{6}{|c|}{ 8B1 (light yellow sands) } & \multicolumn{6}{|c|}{ 12B (yellow sands) } \\
\hline & LY1 & LY2 & LY3 & LY4 & LY5 & LY6 & YS1 & YS2 & YS3 & YS4 & YS5 & YS6 \\
\hline $\mathrm{SiO}_{2}$ & 0.00 & 0.02 & 0.01 & 0.04 & 0.00 & 0.00 & 0.00 & 0.00 & 0.01 & 0.03 & 0.04 & 0.01 \\
\hline $\mathrm{TiO}_{2}$ & 98.46 & 98.13 & 98.88 & 98.35 & 97.68 & 97.77 & 98.31 & 98.32 & 99.12 & 98.37 & 98.09 & 98.92 \\
\hline $\mathrm{Al}_{2} \mathrm{O}_{3}$ & 0.01 & 0.02 & 0.01 & 0.04 & 0.02 & 0.00 & 0.05 & 0.01 & 0.01 & 0.02 & 0.00 & 0.01 \\
\hline $\mathrm{FeO}$ & 0.09 & 0.08 & 0.07 & 0.09 & 0.15 & 0.10 & 0.19 & 0.03 & 0.12 & 0.07 & 0.08 & 0.09 \\
\hline $\mathrm{MnO}$ & 0.00 & 0.01 & 0.04 & 0.00 & 0.04 & 0.00 & 0.02 & 0.01 & 0.03 & 0.03 & 0.00 & 0.05 \\
\hline $\mathrm{MgO}$ & 0.01 & 0.00 & 0.00 & 0.00 & 0.00 & 0.01 & 0.01 & 0.01 & 0.02 & 0.01 & 0.00 & 0.01 \\
\hline $\mathrm{CaO}$ & 0.00 & 0.01 & 0.00 & 0.01 & 0.01 & 0.05 & 0.02 & 0.00 & 0.01 & 0.01 & 0.03 & 0.01 \\
\hline $\mathrm{Na}_{2} \mathrm{O}$ & 0.03 & 0.03 & 0.01 & 0.01 & 0.01 & 0.00 & 0.01 & 0.01 & 0.01 & 0.02 & 0.01 & 0.01 \\
\hline $\mathrm{K}_{2} \mathrm{O}$ & 0.00 & 0.01 & 0.02 & 0.02 & 0.01 & 0.01 & 0.04 & 0.02 & 0.01 & 0.02 & 0.05 & 0.00 \\
\hline $\mathrm{Cr}_{2} \mathrm{O}_{3}$ & 0.32 & 0.39 & 0.07 & 0.06 & 0.21 & 0.23 & 0.20 & 0.23 & 0.22 & 0.06 & 0.90 & 0.14 \\
\hline
\end{tabular}


Table 1 continued

\begin{tabular}{|c|c|c|c|c|c|c|c|c|c|c|c|c|}
\hline \multirow{2}{*}{$\begin{array}{l}\text { Sample no. } \\
\text { Grain no. }\end{array}$} & \multicolumn{6}{|c|}{ 8B1 (light yellow sands) } & \multicolumn{6}{|c|}{ 12B (yellow sands) } \\
\hline & LY1 & LY2 & LY3 & LY4 & LY5 & LY6 & YS1 & YS2 & YS3 & YS4 & YS5 & YS6 \\
\hline $\mathrm{Nb}_{2} \mathrm{O}_{3}$ & 0.20 & 0.21 & 0.01 & 0.12 & 0.53 & 0.30 & 0.61 & 0.01 & 0.00 & 0.03 & 0.30 & 0.30 \\
\hline $\mathrm{Ta}_{2} \mathrm{O}_{5}$ & 0.02 & 0.01 & 0.01 & 0.04 & 0.07 & 0.01 & 0.05 & 0.04 & 0.01 & 0.01 & 0.05 & 0.01 \\
\hline $\mathrm{P}_{2} \mathrm{O}_{5}$ & 0.01 & 0.00 & 0.00 & 0.01 & 0.04 & 0.00 & 0.03 & 0.03 & 0.01 & 0.02 & 0.02 & 0.01 \\
\hline Total & 99.15 & 98.92 & 99.13 & 98.79 & 98.77 & 98.48 & 99.54 & 98.72 & 99.58 & 98.7 & 99.57 & 99.57 \\
\hline \multicolumn{13}{|c|}{ Structural formulae normalized to 4 oxygens } \\
\hline $\mathrm{Si}$ & 0.00 & 0.00 & 0.00 & 0.00 & 0.00 & 0.00 & 0.00 & 0.00 & 0.00 & 0.00 & 0.00 & 0.00 \\
\hline $\mathrm{Al}$ & 0.00 & 0.00 & 0.00 & 0.00 & 0.00 & 0.00 & 0.00 & 0.00 & 0.00 & 0.00 & 0.00 & 0.00 \\
\hline $\mathrm{Ti}$ & 1.99 & 1.99 & 2.00 & 1.99 & 1.98 & 1.99 & 1.98 & 1.99 & 1.99 & 1.99 & 1.98 & 1.99 \\
\hline $\mathrm{Fe}$ & 0.00 & 0.00 & 0.00 & 0.00 & 0.00 & 0.00 & 0.00 & 0.00 & 0.00 & 0.00 & 0.00 & 0.00 \\
\hline $\mathrm{Mn}$ & 0.00 & 0.00 & 0.00 & 0.00 & 0.00 & 0.00 & 0.00 & 0.00 & 0.00 & 0.00 & 0.00 & 0.00 \\
\hline $\mathrm{Mg}$ & 0.00 & 0.00 & 0.00 & 0.00 & 0.00 & 0.00 & 0.00 & 0.00 & 0.00 & 0.00 & 0.00 & 0.00 \\
\hline $\mathrm{Ca}$ & 0.00 & 0.00 & 0.00 & 0.00 & 0.00 & 0.00 & 0.00 & 0.00 & 0.00 & 0.00 & 0.00 & 0.00 \\
\hline $\mathrm{Na}$ & 0.00 & 0.00 & 0.00 & 0.00 & 0.00 & 0.00 & 0.00 & 0.00 & 0.00 & 0.00 & 0.00 & 0.00 \\
\hline $\mathrm{K}$ & 0.00 & 0.00 & 0.00 & 0.00 & 0.00 & 0.00 & 0.00 & 0.00 & 0.00 & 0.00 & 0.00 & 0.00 \\
\hline $\mathrm{Cr}$ & 0.01 & 0.01 & 0.00 & 0.00 & 0.00 & 0.00 & 0.00 & 0.00 & 0.00 & 0.00 & 0.02 & 0.00 \\
\hline $\mathrm{Nb}$ & 0.00 & 0.00 & 0.00 & 0.00 & 0.01 & 0.00 & 0.01 & 0.00 & 0.00 & 0.00 & 0.00 & 0.00 \\
\hline $\mathrm{Ta}$ & 0.00 & 0.00 & 0.00 & 0.00 & 0.00 & 0.00 & 0.00 & 0.00 & 0.00 & 0.00 & 0.00 & 0.00 \\
\hline $\mathrm{P}$ & 0.00 & 0.00 & 0.00 & 0.00 & 0.00 & 0.00 & 0.00 & 0.00 & 0.00 & 0.00 & 0.00 & 0.00 \\
\hline Total & 2.00 & 2.00 & 2.00 & 2.00 & 2.00 & 2.00 & 2.00 & 2.00 & 2.00 & 2.00 & 2.01 & 2.00 \\
\hline
\end{tabular}
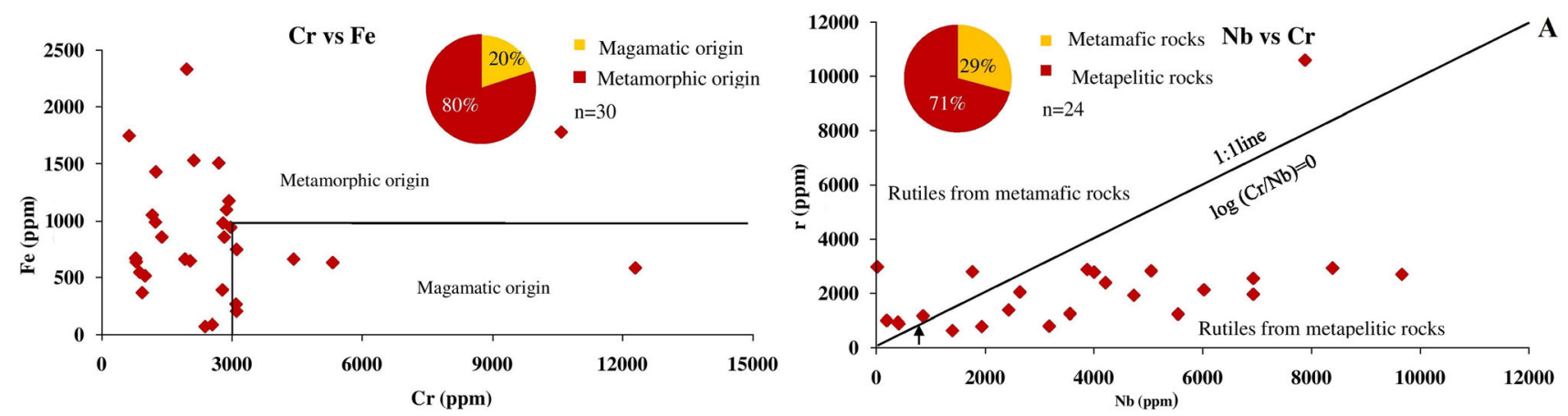

$\mathrm{Cr}$ vs Fe

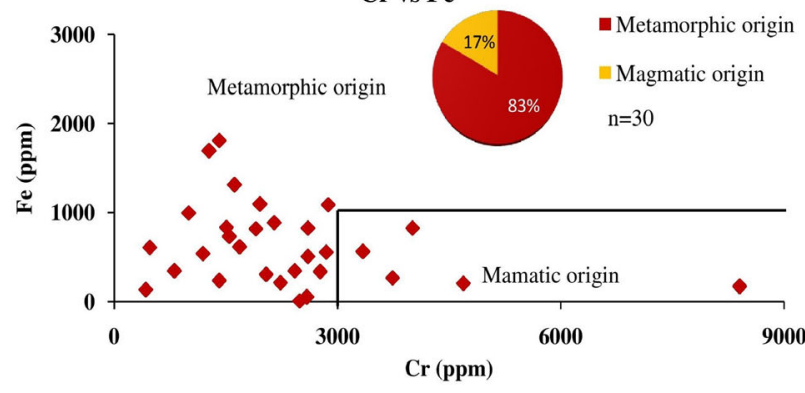

$\mathrm{Nb}$ vs $\mathrm{Cr}$

B

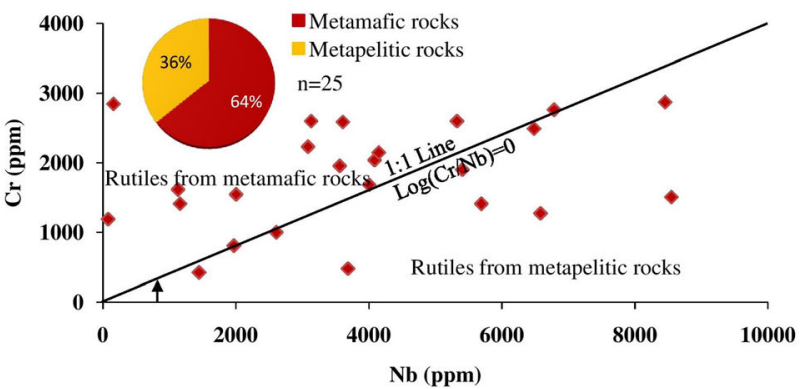

Fig. 2 Scatter plots of $\mathrm{Cr}$ versus $\mathrm{Fe}$ (Zack et al. [8,9]) and $\mathrm{Nb}$ versus $\mathrm{Cr}$ (after Triebold et al. [21]) contents of rutiles. a Red sediments and b modern coastal sands of Bhimunipatnam-Konada coast

$\mathrm{Al}$ and $\mathrm{Mg}$ behavior in rutile has been used to discriminate those derived from crustal and mantle origin [10]. The $\mathrm{Al}$ and $\mathrm{Mg}$ scatter diagrams (Fig. 3a, b) show that most of the rutiles are from crustal rocks.
- Rutiles with $\mathrm{Cr}$ content less than $3000 \mathrm{ppm}$ and $\mathrm{Cr}$ versus $\mathrm{Fe}$ scatter plot suggest that they are derived from metamorphic rocks with an insignificant contribution from magmatic rocks. 


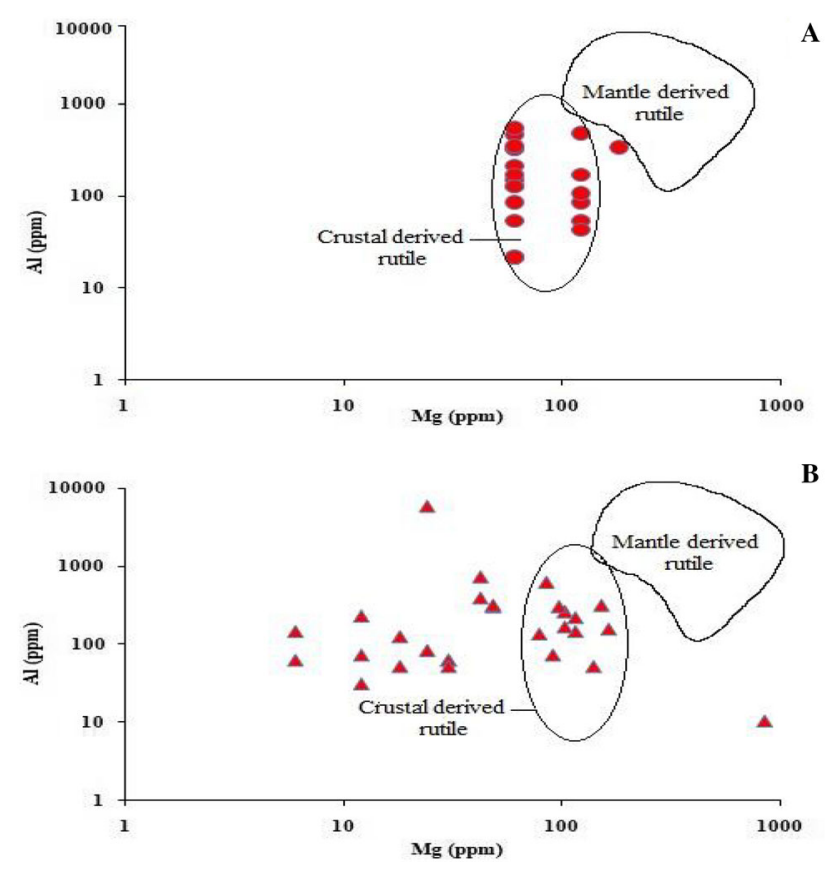

Fig. 3 Scatter plots of $\mathrm{Mg}$ versus $\mathrm{Al}$ contents of rutiles from a red sediments and $\mathbf{b}$ modern coastal sands of Bhimunipatnam-Konada

- $\mathrm{Nb}$ versus $\mathrm{Cr}$ scatter plots for rutiles suggest that they are derived mainly from metapelitic rocks in the study area.

- The $\mathrm{Al}$ and $\mathrm{Mg}$ behavior in rutile from red sediments and modern sands of Bhimunipatnam-Konada coast shows that most of the rutiles are derived from crustal rocks.

- The geochemical behavior of rutile from red sediments and modern sands of Bhimunipatnam-Konada coast indicates that the provenances of rutile for both are unique.

Acknowledgements We thank the authorities of Department of Geology, Andhra University, Visakhapatnam and Geological Survey of India, Hyderabad, for providing laboratory facilities (CAMECA SX-100 electron probe microanalyzer) to carry out geochemical analysis, and CSIR, New Delhi for financial assistance to K. Bangaku Naidu in the form of SRF.

Open Access This article is distributed under the terms of the Creative Commons Attribution 4.0 International License ( http://creativecommons.org/licenses/by/4.0/), which permits unrestricted use, distribution, and reproduction in any medium, provided you give appropriate credit to the original author(s) and the source, provide a link to the Creative Commons license, and indicate if changes were made.

\section{References}

1. Gotaze J (1996) Genetic information of accessory minerals in clastic sediments. Zentralbl Geol Palaontol Teil 1(1995):101-118
2. Deer WA, Howie RA, Zussman J (1992) An Introduction to the rock-forming minerals, 2nd edn. Pearson Education Ltd, Harlow

3. Force ER (1980) The Provenance of rutile. Sed Petro 50:485-488

4. Force ER (1991) Geology of titanium-mineral deposits, Geological Society of America. Special Paper. vol 259, pp 1-112

5. Preston J, Hartley A, Mange-Rajetzky M, Hole MJ, May G, Buck $S$ (2002) The provenance of Triassic continental sandstones from the Beryl Field, northern North Sea: mineralogical, geochemical, and sedimentological constraints. J Sediment Res 72:18-29

6. Preston RJ, Hartley A, Hole MJ, Buck S, Bond J, Mange-Rajetzky M, Still J (1998) Integrated whole-rock trace element geochemistry and heavy-mineral chemistry studies: aids to the correlation of continental red-bed reservoirs in the Beryl Field, U.K. North Sea. Pet Geosci 4:7-16

7. Zack T, Kronz A, Foley S, Rivers T (2002) Trace element abundances in rutiles from eclogites and associated garnet mica schists. Chem Geol 184:97-122

8. Zack T, Moraes R, Kronz A (2004) Temperature dependence of $\mathrm{Zr}$ in rutile: empirical calibration of a rutile thermometer. Contrib Mineral Petrol 148:471-488

9. Zack T, Eynatten VH, Kronz A (2004) Rutile geochemistry and its potential use in quantitative provenance studies. J Sediment Geol 171:37-58

10. Smythe DJ, Schulze DJ, Brenan JM (2008) Rutile as a kimberlite indicator mineral: minor and trace element geochemistry. In: 9th international kimberlite conference extended. Abstract, No 9IKCA-00193

11. Luvizotto LG, Zack $\mathrm{T}$ (2009) $\mathrm{Nb}$ and $\mathrm{Zr}$ behavior in rutile during high-grade metamorphism and retrogression: an example from the Ivrea-Verbano Zone. Chem Geol 261:302-317

12. Morton A, Chenery S (2009) Detrital rutile geochemistry and thermometry as guides to provenance of Jurassic-Paleocene Sandstones of the Norwegian Sea. J Sediment Res 79:540-553

13. Meinhold G, Anders B, Kostopoulos D, Reischmann T (2008) Rutile chemistry and thermometry as provenance indicator: an example from Chios Island, Greece. Sediment Geol 203:98-111

14. Meinhold G (2010) Rutile and its applications in earth sciences. Earth Sci Rev 102:1-28

15. Kanouo SN, Yongue FR, Chen S, Njonfang E, Ma Ch, Ghogomu TR, Zhao J, Sababa E (2012) Greyish-black rutile megaclasts from the Nsanaragati gem placer, SW Cameroon: geochemical features and genesis. J Geogr Geol 4(2):134-146

16. Stendal H, Toteu FS, Frei R, Penaye J, Njel OU, Bassahak J, Nni J, Kankeu B, Ngako V, Hell VJ (2006) Derivation of detrital rutile in the Yaounde' region from Cameroon the Neoproterozoic Pan-African belt in southern Cameroon (Central Africa). J Afr Earth Sci 44:443-458

17. Triebold S, Eynatten VH, Luvizotto LG, Zack T (2007) Deducing source rock lithology from detrital rutile geochemistry: example from the Erzgebirge, Germany. Chem Geol 244:421-436

18. Hubert JF (1962) Zircon-Tourmaline-Rutile maturity index and the interdependence of the composition of heavy mineral assemblages with the gross composition and texture of sandstones. J Sediment Petrol 32(3):440-450

19. Morton AC, Halls Worth CR (1999) Processes controlling the composition of detrital heavy mineral assemblages in sandstones. Sediment Geol 124:3-29

20. Morton AC, Hallsworth CR (2007) Stability of detrital heavy minerals during burial diagenesis. In: Mange M, Wright DT (eds) Heavy minerals in use, vol 58. Elsevier, Amsterdam, pp 215-245

21. Triebold S, Von Eynatten H, Zack T (2005) Trace elements in detrital rutile as provenance indicator: A case study from the Erzgebirge, Germany. In: Haas H, Ramseyer K, Schlunegger F (eds) Abstracts. Schriftenr, vol 38. Dt Ges Geowiss, Schriftenr, pp 144-145 
22. Subramanyam NP, Rao GVU (1980) Niobium and tantalum in the rutile of Kerala beach sands. J Geol Soc India 21:623-626

23. Kamineni DC, Rao AT (1988) Sapphirine-granulites from the Kakanuru area, Eastern Ghats, India. Am Mineral 73:692-700

24. Divakara Rao V (1984) Khondalites from the Eastern Ghats granulite belt, geochemistry and origin. Geophys Res Bull 21(3):233-242

25. Leelanandam C (1990) The anorthosite complexes and Proterozoic Mobile Belt of Peninsular India. In: Naqvi SM (ed) Precambrian continental crust and its economic mineral resources; development in Precambrian geology, vol 8. Elsevier, Amsterdam, pp 409-436

26. Narayana BL, Rama Rao P, Reddy GLN, Divakara Rao V (1995) Geochemistry and origin of megacrystic charnockites and granites from Eastern Ghat Granulite Belt. In: Proceedings of symposium on India and Antarctica during the Precambrian and granulite and crustal processes in East Gondwana; Dec. 1-3, Andhra University, Visakhapatnam, p 36
27. Rudnick LR, Barth M, Horn I, McDonough FW (2000) Rutilebearing refractory eclogites: missing link between continents and depleted mantle. Science 287:278-281

28. Hedge VS, Shalini G, Kanchanagouri DG (2006) Provenance of heavy minerals with special reference to ilmenite of the Honnavar beach, central west coast of India. Curr Sci 91(5):644-648

29. Plank T, Langmuir CH (1998) The chemical composition of subducting sediment and its consequences for the crust and mantle. Chem Geol 145:325-394

30. Barth MG, Mc Donoghue WF, Rudnick RL (2000) Tracking the budget of $\mathrm{Nb}$ and $\mathrm{Ta}$ in the continental crust. Chem Geol 165:197-213

Publisher's Note Springer Nature remains neutral with regard to jurisdictional claims in published maps and institutional affiliations. 